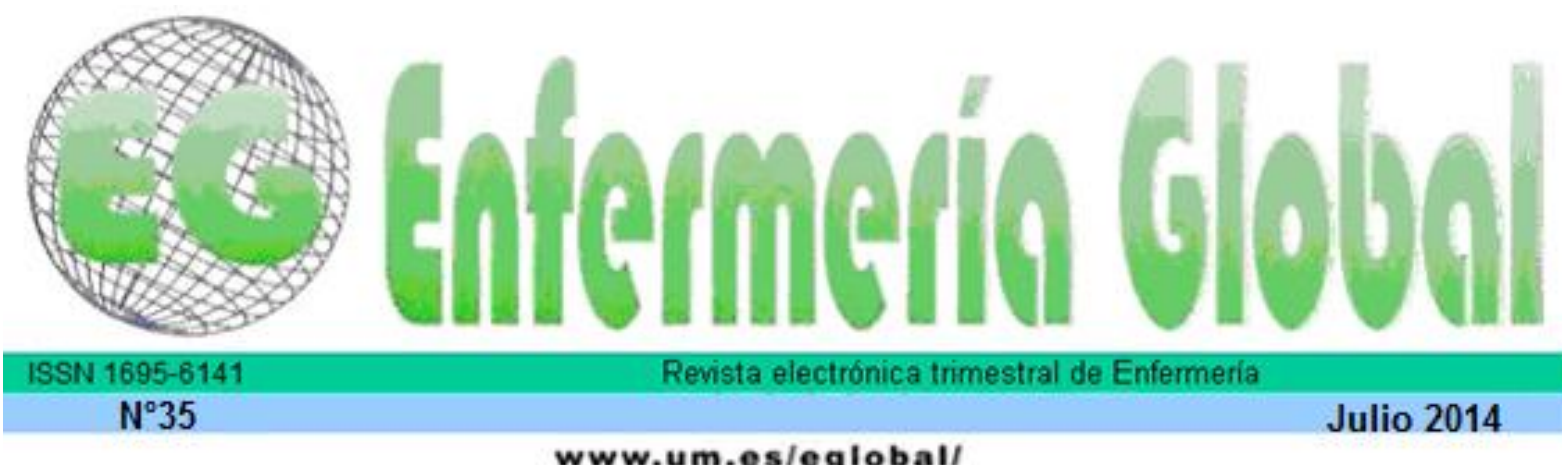

www.um.es/egloball

\title{
CLÍNICA
}

\section{Modelos lineales de temperatura corporal en el paciente postquirúrgico}

Body temperature linear models in postsurgical patients

\section{*Izquierdo Monge, Dolores *Beltrán Guerra, Isabel **Santos, José Manuel ${ }^{* * *}$ Ortega Calvo, Manuel}

\begin{abstract}
*DUE. Unidad de Recuperación Postanestésica. Hospital Universitario Virgen Macarena. E-mail: Iolaizquierdo18@gmail.com **Médico de Familia. Unidad de Investigación. Distrito Sanitario de Atención Primaria. CIBER - OBN. * ****Médico de Familia. Unidad de Investigación. Distrito Sanitario
\end{abstract} de Atención Primaria. ISCIII. Sevilla.

Palabras clave: temperatura corporal; período de recuperación de la anestesia; enfermería postanestésica; modelos lineales; modelos logísticos.

Keywords: body temperature, anesthesia recovery period, postanesthesia nursing, linear models, logistic models.

\section{RESUMEN}

Introducción y objetivos: La hipotermia no intencionada es una situación ante la cual la enfermería ha de estar entrenada para su reconocimiento y control. Nuestros objetivos han sido: evaluar la proporción de pacientes que llegaban en situación de hipotermia (temperatura $<36 \circ \mathrm{C}$ ) y estudiar el comportamiento de la temperatura corporal en la Unidad de Recuperación Postanestésica (URPA).

Material y métodos: La medición se llevó a cabo mediante un termómetro timpánico electrónico infrarrojo. El contraste de medias aritméticas se realizó mediante pruebas T. Cuando las variables contenían más de dos categorías se empleó la ANOVA de un factor. Se construyeron modelos con regresión lineal y logística.

Resultados: El 85,26\% de los pacientes presentaron hipotermia. La temperatura media de ingreso de los pacientes tratados con anestesia combinada fue significativamente más baja que la de los tratados con local o general. Tanto en los modelos univariantes como en los multivariantes con regresión lineal, la temperatura medida a los 90 minutos fue la que más se correlacionó con la temperatura al alta (coeficiente de determinación $\mathrm{R}^{2}=0.69 ; \mathrm{p}<0.001$ ). 
Discusión: Hemos encontrado una proporción elevada de pacientes con hipotermia (temperatura corporal menor de $\left.36^{\circ} \mathrm{C}\right)$ a su ingreso en la URPA $(85,26 \%)$. No hemos encontrado ninguna variable predictora para la hipotermia de llegada a pesar de que los pacientes anestesiados de manera combinada ingresaban con una temperatura media menor que aquellos tratados con local o con general (ANOVA de un factor $p<0.05$ ). Consideramos una estancia mínima de 90 minutos para un control adecuado de la variable temperatura en un entorno de recalentamiento empírico no protocolizado.

\section{ABSTRACT}

Introduction and objectives: unintentional hypothermia is a situation in which nursing has to be trained for recognition and control. Our objectives were to evaluate the proportion of patients arriving in situations of hypothermia (temperature $<36{ }^{\circ} \mathrm{C}$ ) and to study the behavior of body temperature in the post-anesthesia care unit.

Methods: the measurement was carried out by infrared electronic tympanic thermometer. The contrast arithmetic test was performed by $T$ tests. When variables contained more than two categories, we used the one-way ANOVA. Models were constructed with linear and logistic regression.

Results: $85.26 \%$ of patients had hypothermia. The average admission temperature of patients treated with combined anesthesia was significantly lower than that of those treated with local or general. Both in the univariate and in multivariate models using linear regression, the temperature measured at 90 minutes was the most correlated with the temperature at discharge (coefficient of determination R2 = $0.69, \mathrm{P}<0.001)$.

Discussion: we found a high proportion of patients with hypothermia (body temperature below $36^{\circ} \mathrm{C}$ ) on admission (85.26\%). We found no predictor for arrival hypothermia despite combined anesthetized patients were admitted with an average temperature measuring less than those treated with local or general (one-way ANOVA $p$ <.05). We consider a minimum stay of 90 minutes for proper control of the temperature variable in a non docketed empirical overheating

\section{INTRODUCCIÓN}

La temperatura es una de las variables más importantes para el control postanestésico correcto. La hipotermia no intencionada es una situación ante la cual la enfermería ha de estar especialmente entrenada ${ }^{1}$ para su reconocimiento y control. Entre los efectos de la hipotermia sobre el sistema cardiovascular merecen señalarse los siguientes: disminución del gasto cardíaco, descenso de la frecuencia cardíaca y de la presión arterial, trastornos en la conducción miocárdica y arritmias, angina postoperatoria, aumento de la resistencia vascular periférica y disminución de la capacitancia venosa ${ }^{2}$. Entre los pacientes que tienen más riesgo de sufrir una hipotermia postanestésica deben citarse: niños, ancianos, quemados, traumatizados y desnutridos ${ }^{3}$.

Durante la fase perioperatoria, los pacientes pueden presentar hipotermia por los mecanismos siguientes: radiación, conducción, convección y evaporación. Debemos citar también el fenómeno de la redistribución de la sangre periférica fría con la central más caliente que tiene lugar durante la inducción anestésica.

Algunos sedantes como el propofol, los anestésicos inhalados como el óxido nitroso y los miorrelajantes disminuyen la vasoconstricción y aumentan la hipotermia; cerrando el círculo, la hipotermia prolonga el efecto de estos fármacos.

Se han diseñado también diferentes estrategias de recalentamiento en estos pacientes ${ }^{4}$. Se pueden clasificar en técnicas de recalentamiento activo y en técnicas de recalentamiento pasivo ${ }^{5}$. La causa principal de la hipotermia en la anestesia 
regional, es el bloqueo nervioso periférico, mientras que en la anestesia general lo es la alteración del centro regulador del hipotálamo².

Teniendo todo ello en cuenta, nuestro objetivos en este trabajo han sido: 1) Evaluar la proporción de pacientes que llegaban en situación de hipotermia (temperatura $<36^{\circ} \mathrm{C}$ ) después de una intervención quirúrgica reglada, y 2) Estudiar el comportamiento de la temperatura corporal medida mediante modelos de regresión lineal y logística binaria en los pacientes ingresados en la URPA (Unidad de Recuperación Postanestésica) del hospital terciario en donde trabajamos a los que se aplicaron técnicas de recalentamiento tanto activas como pasivas de forma empírica y sin un protocolo predeterminado.

\section{MATERIAL Y MÉTODOS}

Para el análisis mediante regresión lineal, calculamos el tamaño de muestra con el programa GRANMO versión 7.12 del Instituto Hospital del Mar de Investigaciones Médicas (IMIM) ${ }^{6}$. Los tamaños muestrales oscilaban entre 25 y 107 pacientes contemplando un $20 \%$ de pérdidas y unos coeficientes de correlación $(R)$ entre 0.6 y 0.3 . Todo ello con un error alfa del $5 \%$ y con una potencia del $80 \%$. (Tabla 1 ). Aunque en el software no viene descrito, entendimos que estos valores son aplicables a la situación de una sola predictora en el modelo de regresión lineal. Los pacientes fueron seleccionados de forma aleatoria por conveniencia cuando ingresaban en la URPA durante un período de tres meses.

Tabla 1: Tamaños muestrales calculados con un error alfa del $5 \%$, un error beta del $20 \%$, para un contraste de hipótesis bilateral y con porcentaje de pérdidas en el seguimiento del 20\% (Programa GRANMO - Coeficientes de Correlación).

\begin{tabular}{|l|l|}
\hline $\begin{array}{l}\text { Coeficiente de } \\
\text { correlación. R. }\end{array}$ & $\begin{array}{l}\text { Tamaño de } \\
\text { muestra. }\end{array}$ \\
\hline 0.6 & 25 \\
\hline 0.5 & 37 \\
\hline 0.4 & 59 \\
\hline 0.3 & 107 \\
\hline
\end{tabular}

Se aplicaron criterios de inclusión (intervenciones pertenecientes a las especialidades de cirugía general, traumatología, urología, obstetricia y ginecología) y de exclusión (cirugía de urgencias, cirugía laparoscópica y pacientes pediátricos). Toda la información se recogió en las variables: edad (cuantitativa continua), sexo (categórica) y especialidad quirúrgica (categórica). Se recogió también el tipo de anestesia empleado (Local, General, Combinada) (variable categórica) y las horas de duración de la intervención (menos de una hora, más de una hora, más de dos horas y más de tres horas) (variable categórica).

Para obtener nuestros objetivos, primero teníamos que saber el porcentaje de pacientes que llegaban a la unidad en situación de hipotermia (variable temperatura al ingreso) y el comportamiento posterior de la misma (temperatura a los 30 minutos y a los 90 minutos y temperatura al alta). La medición se realizó mediante un termómetro timpánico electrónico infrarrojo GENIUS $2 \AA$, que mide valores de temperatura muy próximos a la temperatura central. Las mediciones se realizaron por personal entrenado, siempre en el mismo canal auditivo del paciente y con un dígito decimal ${ }^{7}$. La calibración se llevó a cabo siguiendo las normas del fabricante. Consideramos 
como valores de normotermia $36-37^{\circ} \mathrm{C}$ y como valores de hipotermia la temperatura que estuviera por debajo de $36^{\circ} \mathrm{C}$. La variable Hipotermia se diseñó de forma categórica en base a los valores de la temperatura al ingreso (temperatura al ingreso menor a $36^{\circ}$ "Hipotermia Si", resto de valores, "Hipotermia No"). Se construyó un paquete de datos y se analizó con el programa estadístico $R$, en su versión $2.9 .0^{8}$ y el paquete $\mathrm{R}$ Commander. El contraste de medias aritméticas de las variables continuas se realizó mediantes pruebas $T$. Cuando las variables contenían más de dos categorías (por ejemplo: tipo de anestesia) se empleó la ANOVA de un factor. Se plantearon varios modelos multivariantes con regresión lineal para estudiar el comportamiento de la temperatura. La variable dependiente fue la temperatura al alta de la URPA. Las predictoras, la temperatura al ingreso, a los 30 minutos y a los 90 minutos.

La variable Hipotermia en su forma categórica se sometió también a un análisis predictivo mediante regresión logística binaria ${ }^{9} 10$. Las variables independientes fueron la edad, el sexo del paciente, la especialidad de origen, el tiempo de duración de la intervención y el tipo de anestesia y la dependiente el haber presentado hipotermia, no haberla presentado al ingreso en la URPA. Se pidieron los permisos correspondientes al Comité de Ética Local.

\section{RESULTADOS}

Obtuvimos una muestra de 95 pacientes. Los valores perdidos oscilaron entre 1 en la variable sexo y 17 en la variable temperatura a los 90 minutos. No se imputaron valores en las variables cuantitativas y se realizó un análisis sobre la información existente. La muestra estaba compuesta por 36 varones $(38,3 \%)$ y 58 mujeres (61,7\%). La distribución de la variable edad está recogida en la Figura 1.

Figura 1: Diagrama de cajas de la variable edad discriminada por el sexo.

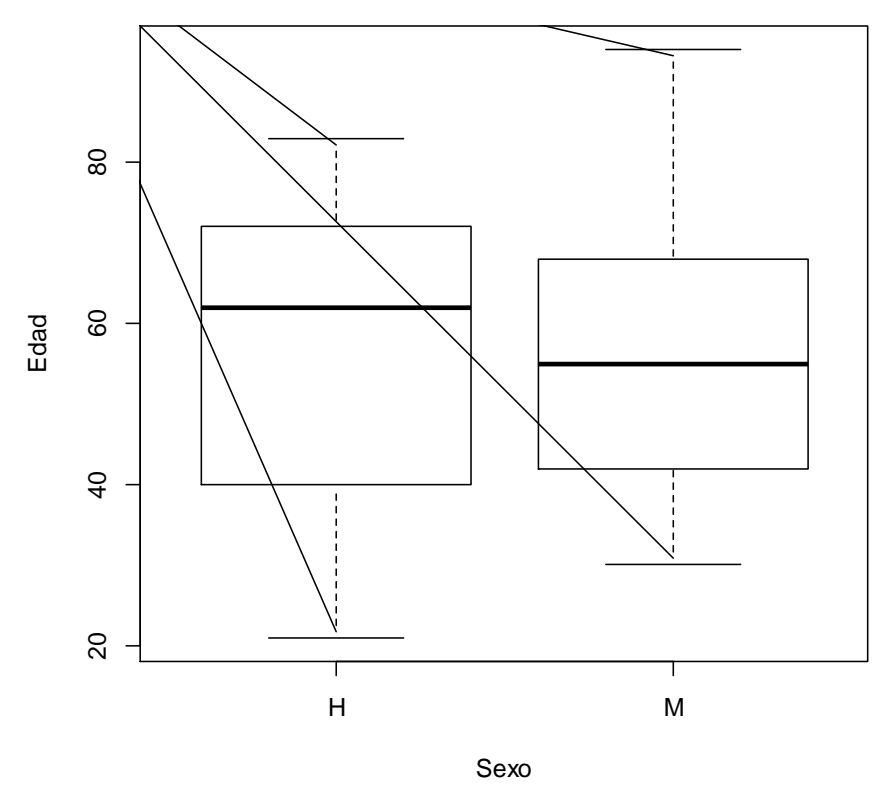

Tanto para los supuestos de varianzas desiguales $(t=0.1351 ; p=0.89)$ como de varianzas iguales ( $\mathrm{t}=0.1373 ; \mathrm{p}=0.89$ ) la comparación de medias aritméticas de los dos 
grupos no arrojó diferencias significativas. El origen de los pacientes está recogido en la Figura ํo 2.

Figura 2: Gráfica de barras de la variable "Especialidad de Origen". (CG= Cirugía General, G=Ginecología, O=Obstetricia, T=Traumatología, U=Urología).

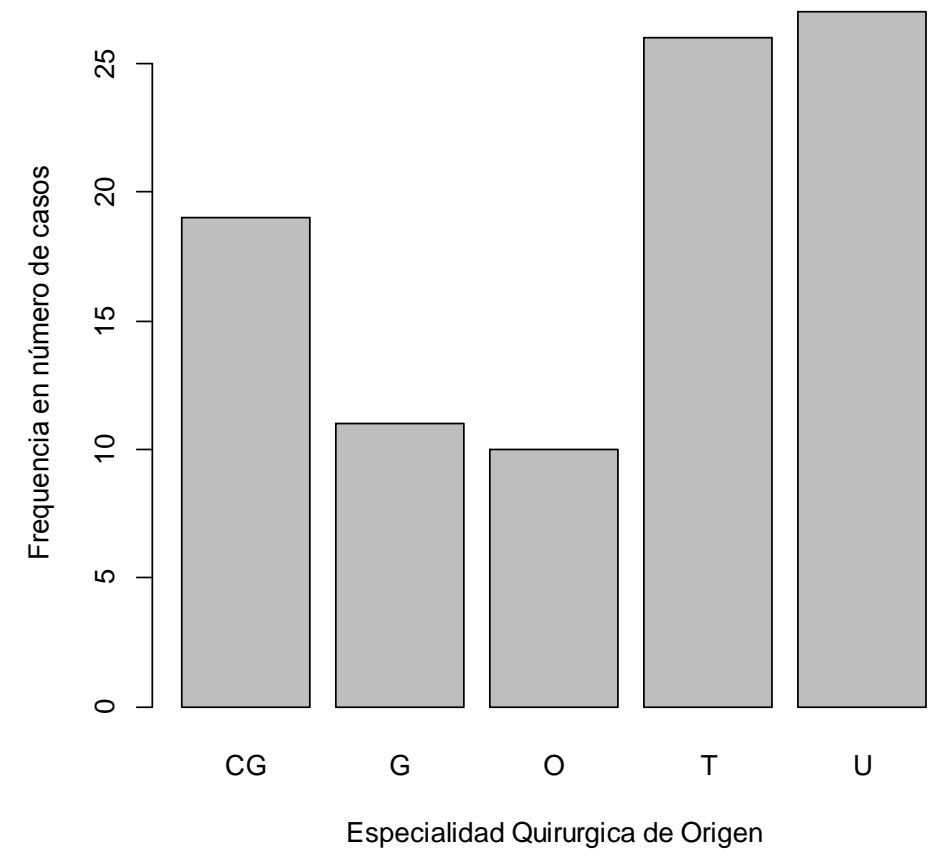

Los tipos de anestesia fueron: local en 45 casos $(49,45 \%)$, general en 28 casos $(30,77 \%)$ y combinada en 18 casos $(19,78 \%)$.

Las gráficas de cajas de las temperaturas al ingreso según las especialidades de origen están recogidas en la Figura 3 y según el tipo de anestesia en la Figura 4.

Figura 3: Temperaturas al ingreso según la especialidad de origen ( $C G=$ Cirugía General, $\mathrm{G}=$ Ginecología, $\mathrm{O}=$ Obstetricia, $T=$ Traumatología, U=Urología). ANOVA de un factor no significativa $(p>0.05)$.

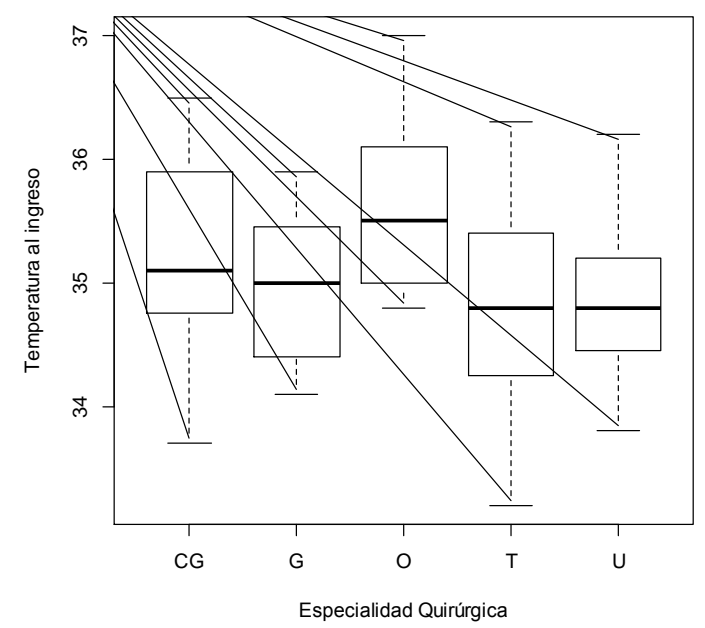


Figura 4: Temperatura al ingreso según el tipo de anestesia empleada $(\mathrm{C}=$ combinada, $G=$ general y $L=$ local)

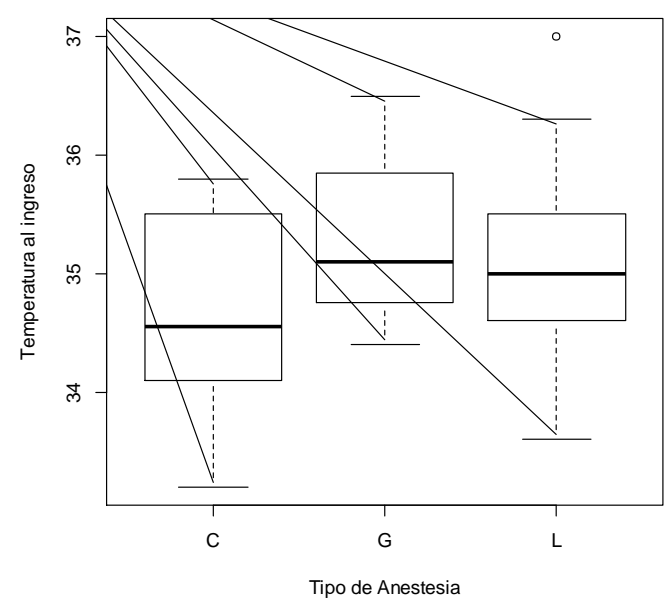

La ANOVA de un factor para la comparación de medias aritméticas de la temperatura al ingreso entre los tres grupos de tipos de anestesia (Figura 4) resultó significativa para la anestesia combinada (media aritmética $=34,68^{\circ} \mathrm{C}$ con una desviación estándar de $0.77, p=0.0339$ ). En la Figura 5 se expone la gráfica de sectores de la temperatura al ingreso categorizada en forma de Hipotermia Sí/No. El 85,26 \% de nuestra muestra ingresó en la URPA en situación de hipotermia.

Figura 5: Gráfica de sectores de la variable Hipotermia (Sí = 81 paciente $-85.26 \%$; No = 14 pacientes - 14.74\%).

Hipotermia

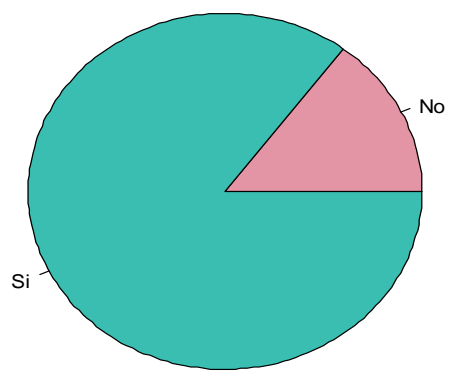

En la Figura 6 se puede observar la distribución del tiempo de intervención. 
Figura 6: Duración de la intervención en la muestra de pacientes estudiados.

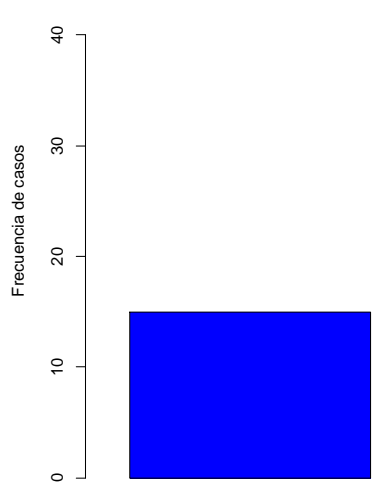

Menos de 1 hora

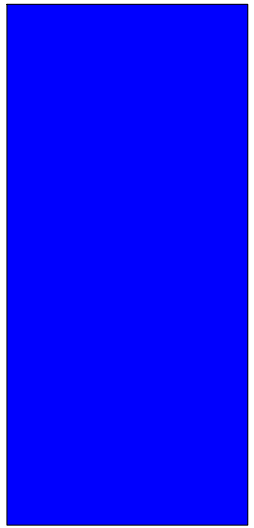

Más de 1 hora

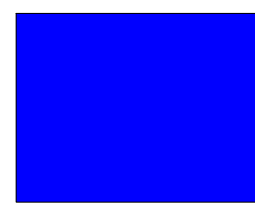

Más de 2 horas

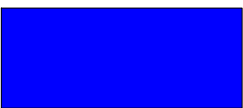

Más de 3 horas

En el análisis mediante regresión logística binaria, ninguna variable (edad, sexo, especialidad quirúrgica de origen, duración de la intervención, tipo de anestesia y tiempo de intervención) se mostró significativamente predictora de hipotermia al ingreso en la URPA.

Después de realizar la estadística descriptiva y el estudio predictivo mediante regresión logística, planteamos varios modelos multivariantes con regresión lineal para estudiar el comportamiento de la temperatura. La variable dependiente fue la temperatura al alta de la URPA. Las predictoras, la temperatura al ingreso, a los 30 minutos y a los 90 minutos. En la Figura 7 se puede observar la matriz de nube de puntos y las rectas de regresión correspondientes de las diferentes temperaturas recogidas en este artículo. En la diagonal de esta figura se pueden observar las gráficas Quantil-Quantil (Q-Q) con respecto a la distribución normal de las diferentes temperaturas observadas. 
Figura 7: Matriz de nubes de puntos de las variables: Temperatura al ingreso, a los 30 minutos, a los 90 minutos y al alta. La diagonal está ocupada por los gráficos cuantil-cuantil (Q-Q) con respecto a la curva normal de cada una de las variables.

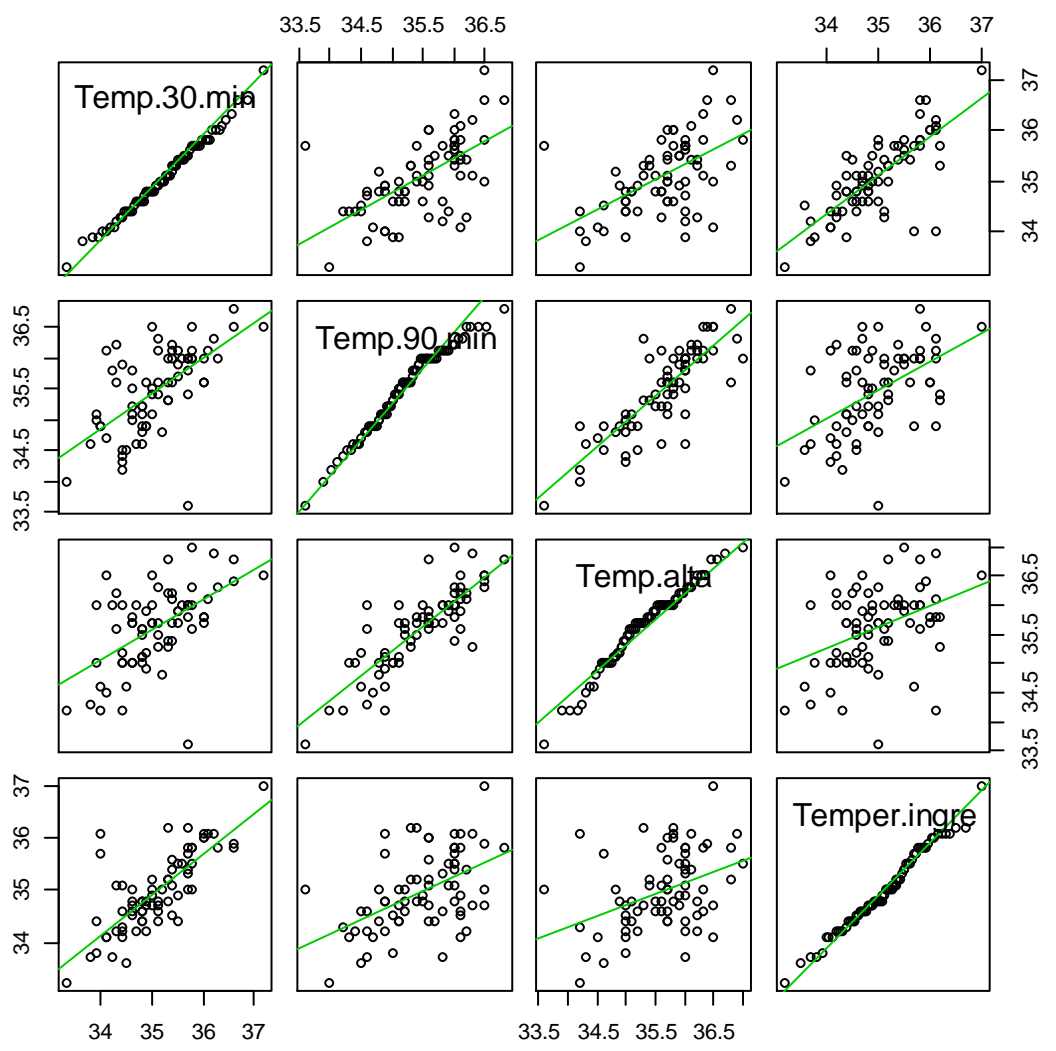

En los tres modelos monovariantes que realizamos tan sólo el que contenía como predictora la temperatura a los 90 minutos tuvo un coeficiente de determinación elevado $\left(R^{2}\right.$ ajustado $\left.=0.69 ; \mathrm{p}<0.001\right)$ (Tabla 2). En los modelos multivariantes realizados con regresión lineal, la variable que más se correlacionó con la temperatura al alta fue también la medida a los 90 minutos de estancia con un $\mathrm{R}^{2}$ de $0.69(p<0.05)$. Las temperaturas al ingreso y a los 30 minutos no resultaron significativas en el análisis multivariante (Tabla 3).

Tabla 2: Estimaciones de modelos con regresión lineal monovariante en el que se consideró la temperatura al alta como variable dependiente y las temperaturas al ingreso, a los 30 y a los 90 minutos como predictoras (tres modelos distintos con una sola predictora).

\begin{tabular}{|l|l|l|l|l|l|}
\hline $\begin{array}{l}\text { Variable } \\
\text { Dependiente } \\
\text { (Y): }\end{array}$ & $\beta_{0}$ & $\beta_{\mathrm{i}}$ & $\begin{array}{l}\text { Valor de } \\
\mathrm{T} \beta_{\mathrm{i}} \\
\text { Temperatura }\end{array}$ & $\begin{array}{l}\text { Significación } \\
\beta_{\mathrm{i}}\end{array}$ & $\mathrm{R}^{2}$ \\
\hline $\begin{array}{l}\text { Temperatura } \\
\text { al ingreso }\end{array}$ & 21.79 & 0.39 & 4.51 & $\mathrm{P}<0.001$ & 0.18 \\
\hline $\begin{array}{l}\text { Temperatura a } \\
\text { los 30' }\end{array}$ & 17.36 & 0.51 & 6.30 & $\mathrm{P}<0.001$ & 0.31 \\
\hline $\begin{array}{l}\text { Temperatura a } \\
\text { los 90' }\end{array}$ & 5.68 & 0.84 & 13.05 & $\mathrm{P}<0.001$ & 0.69 \\
\hline
\end{tabular}


Tabla 3: Estimaciones del modelo con regresión lineal multivariante en el que se consideró la temperatura al alta como variable dependiente y las temperaturas al ingreso, a los 30 y a los 90 minutos como predictoras. $\mathrm{R}^{2}$ ajustado $=0.6915$ (un solo modelo multivariante con tres predictoras).

\begin{tabular}{|c|c|c|c|c|}
\hline $\begin{array}{ll}\beta_{0} & y \\
\text { variables }\end{array}$ & Estimación & $\begin{array}{l}\text { Error } \\
\text { Estándar }\end{array}$ & Valor de T & Significación \\
\hline$\beta_{0}$ & 5.72 & 2.62 & 2.18 & 0.03 \\
\hline $\begin{array}{l}\text { Temperatura } \\
\text { al ingreso }\end{array}$ & -0.12 & 0.09 & -1.27 & 0.20 \\
\hline $\begin{array}{l}\text { Temperatura } \\
\text { a los } 30^{\prime}\end{array}$ & 0.14 & 0.10 & 1.42 & 0.15 \\
\hline $\begin{array}{l}\text { Temperatura } \\
\text { a los } 90^{\prime}\end{array}$ & 0.81 & 0.085 & 9.54 & $<0.0001$ \\
\hline
\end{tabular}

Finalmente presentamos un gráfico tridimensional del modelo con dos predictoras (Temperatura al ingreso y a los 90 minutos en los ejes coordenadas) y la temperatura al alta como variable resultado (eje y) (Figura 6).

Figura 6: Representación tridimensional del modelo con dos predictoras (temperatura al ingreso y a $90^{\prime}$. Las escalas de los ejes oscilan entre 30 y $40^{\circ} \mathrm{C}$. El elipsoide central recoge el $50 \%$ de los datos. La variable resultado (eje $\mathrm{Y}$ ) es la temperatura al alta. $R^{2}$ ajustado $=0.68$.

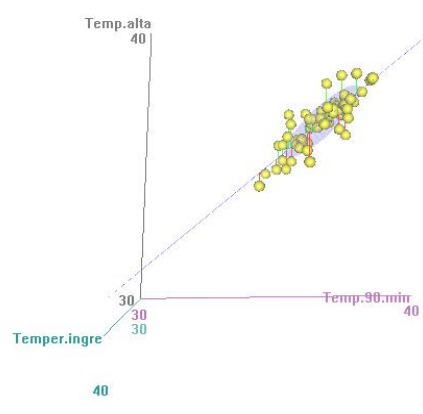

\section{DISCUSIÓN}

Hemos realizado un estudio descriptivo de la hipotermia no intencionada existente en una muestra aleatoria de pacientes ingresados en la URPA multidisciplinaria de un hospital de tercer nivel. Posteriormente hemos realizado un estudio analítico buscando variables predictoras de hipotermia y estudiando el comportamiento lineal de la temperatura del paciente postquirúrgico. No hemos encontrado variables significativamente predictoras de la hipotermia a pesar de manejar un tamaño muestral adecuado y con una tasa de eventos de interés por variable aceptable ${ }^{11}$. 
Queremos señalar en este sentido, el excelente trabajo de Rincón y cols ${ }^{12}$ quienes manejando un tamaño de muestra mayor, encontraron que la edad, el peso, la duración estimada de la intervención y las temperaturas ambiental y corporal durante la fase de inducción de la anestesia resultaron ser predictoras de hipotermia utilizando modelos de regresión logística.

El porcentaje de hipotermia de los pacientes al ingreso se elevó a un 85,26\% (Figura 5). Los porcentajes oscilan en la literatura desde un $41 \%{ }^{13}$ hasta un $93 \%{ }^{14}$. Entendemos que se trata de un problema prevalente ${ }^{15}$ en el cuidado temprano del paciente postquirúrgico.

Entre nuestros resultados merece destacarse la temperatura media de ingreso de los pacientes tratados con anestesia combinada que fue significativamente menor que los tratados sólo con anestesia local o general (ANOVA de un factor $p<0.05$, Figura 4).

En los modelos de regresión lineal realizados para conocer el comportamiento de la temperatura, destacó el que contenía la temperatura medida a los 90 minutos del ingreso. El coeficiente de determinación oscilaba sobre 0.7 , lo cual es un resultado muy bueno (Tabla 3 y Figura 6 ). El $R^{2}$ es tan importante en regresión lineal multivariante que conforma algún que otro aforismo estadístico ${ }^{16}$. Por ejemplo "en regresión lineal, el coeficiente de determinación, $\mathrm{R}^{2}$, va a Misa ¡".

No se midió el tiempo completo de estancia de cada uno de los pacientes, pero la temperatura medida a los 90 minutos fue la que más se correlacionó con la temperatura al alta. Las maniobras de recalentamiento fueron empíricas y no protocolizadas. El alta de la URPA era una decisión del anestesista, que tenía en cuenta la temperatura pero también muchas otras variables clínicas que no analizamos en este artículo. El tiempo de estancia en la unidad postanestésica es un tema de discusión al que deseamos aportar conocimiento con este trabajo ${ }^{17}$.

Al ser esta una investigación cuantitativa no se midieron las sensaciones subjetivas de calor o frío del paciente a su entrada en la URPA. Tampoco se midió la existencia de escalofríos. Las gráficas Q-Q (cuantil-cuantil) mostradas en la diagonal de la Figura 7 muestran una distribución muy cercana a la normal en todas las variables que estudiaban la temperatura de nuestra muestra. Ante esta evidencia visual cualitativa y con el tamaño de muestra que manejábamos ( $n=95$ pacientes) no realizamos pruebas de hipótesis de normalidad.

La diferencia en $\mathrm{R}^{2}$ de la Tabla 3 y de la Figura 8 se debe en que en la primera se incluye un modelo con tres predictoras y en la segunda un modelo con sólo dos. En los diversos modelos analizados con una sola predictora, el que mostraba un coeficiente de determinación más elevado era el que se construía con la temperatura a los 90 minutos (Tabla 2).

En síntesis, hemos encontrado una proporción elevada de pacientes con hipotermia (temperatura corporal menor de $36^{\circ} \mathrm{C}$ ) a su ingreso en la URPA $(85,26 \%)$ concordante con lo descrito por diversos investigadores. No hemos localizado ninguna variable predictora para la hipotermia de llegada a la URPA a pesar de que los pacientes anestesiados de manera combinada ingresaban con una temperatura medía menor que aquellos tratados con local o con general (ANOVA de un factor $p<0.05$ ). Consideramos una estancia mínima de 90 minutos en la URPA multidisciplinaria para 
un control adecuado de la variable temperatura en un entorno de recalentamiento empírico no protocolizado.

\section{Agradecimientos}

Los autores expresan su agradecimiento a Dña. Concepción Lagoa Elías, Responsable de cuidados de la Unidad durante el período en que se realizó este estudio.

\section{BIBLIOGRAFÍA}

1 Fernández-Meré LA, Álvarez-Blanco M: Manejo de la Hipotermia Perioperatoria. Rev Esp Anestesiol Reanim. 2012; 59 : 379-389.

2 Burns SM, Wojnakowski M, Piotrowski K, Caraffa G: Unintentional hypothermia: implications for perianesthesia nurses. J Perianesth Nurs. 2009 ;24:167-73.

${ }^{3}$ Weirich TL : Hypothermia/warming protocols: why are they not widely used in the OR? AORN J. 2008; 87 : 333-44.

4 Pikus E, Hooper V D: Postoperative rewarming: are there alternatives to warm hospital blankets. J Perianesth Nurs. 2010 ;25:11-23.

${ }^{5}$ Noble KA : Chill can kill. J Perianesth Nurs. 2006 ; 21 : 204-207.

6 http://www.imim.es/ofertadeserveis/software-public/granmo/ visitado el 14 de febrero de 2012.

7 Fetzer SJ, Lawrence A : Tympanic membrane versus temporal artery temperatures of adult perianesthesia patients. J Perianesth Nurs. 2008 ; 23 :230-236.

8 Ihaka, R, Gentleman, R R : A language for data analysis and graphics. Journal of computational and graphical statistics.1996; 5:299-314.

9 Sánchez-Cantalejo, E: Regresión logística en Salud Pública. Escuela andaluza de salud pública. Granada. 2000. disponible en :

http://www.easp.es/publicaciones/descargas/easp regresion\%20logistica\%20en\%20s alud\%20publica.pdf

${ }^{10}$ Silva-Ayçaguer, LC, Barroso, IM: Regresión logística. ed. La Muralla. Madrid. 2004.

11 Ortega-Calvo, M, Cayuela-Domínguez, A: Regresión logística binaria y tamaño de muestra : una revisión bibliográfica. rev esp salud publica. 2002 ; 76:85-93. disponible en : http://scielo.isciii.es/pdf/resp/v76n2/a02v76n2.pdf

12 Rincón DA, Valero JF, Eslava-Schmalbach J : Construcción y validación de un modelo predictivo de hipotermia intraoperatoria. Rev Esp Anestesiol Reanim. 2008; 55:401-6.

13 Karalapillai D, Story DA, Calzavacca P, Licari E, Liu YL, Hart GK :Inadvertent hypothermia and mortality in postoperative intensive care patients: retrospective audit of 5050 patients. Anaesthesia. 2009 ;64:968-72.

14 Gómez Martín A, Canseco Hernández C, Tovar Benito D, Delgado Tejedor P, Blanco Guillén A, Ruiz Muñoz Y, Fernández Gómez T, Marín Gallardo C, Sánchez Pérez S, Ureña Romero AM: Hipotermia postquirúrgica: el auxiliar de enfermería asegurando el bienestar y el confort del paciente. Enferm Clin. $2009 ; 19: 48-51$.

15 Abelha FJ, Castro MA, Neves AM, Landeiro NM, Santos CC: Hypothermia in a surgical intensive care unit. BMC Anesthesiol. 2005 Jun 6; 5 :7. Disponible en : http://www.ncbi.nlm.nih.gov/pmc/articles/PMC1180426/pdf/1471-2253-5-7.pdf 
${ }^{16}$ González-García I, Chemello C, García-Sánchez F et al: Aphorisms and short phrases as pieces of knowledge in the pedagogical framework of the andalusian school of public health. int j prev med. $2012 ; 3: 197-210$.

${ }^{17}$ Panagiotis K, Maria P, Argiri P, Panagiotis S : Is postanesthesia care unit length of stay increased in hypothermic patients? AORN J. 2005; $81: 379-82$. 